\title{
Applications and Influence of Intelligent Robot in Education
}

\author{
Ningxian Zhu \\ Shandong University of political science and law \\ Shandong, Jinan, 250014, china \\ zhuningixan@163.com
}

Keywords: Intelligent robot. Intelligent Education. Applying Patterns.

Abstract. At present, Lee Se-dol combats with AlphaGo, it is a historical landmark, and stimulates people to discuss on affection of intelligent robot. They think intelligent robot will trigger a new revolution in the service industry. Similarly, it will have a profound impact on education. This article discusses robot's applications in all walks of life, combined with the characteristics of the application and exists problems in traditional education, discovers its applying patterns and strategy of the intelligent robot, further explores the solutions to intelligent robot in education.

\section{Introduction}

On April 2016, Goldman thinks the VR and AR Will be the next big computing platform, which will be have a profound influence as a computer do. Further, Helmet-mounted display is completely different form PC. It has a better display effect. There are many AR and VR's application in all walks of life. For example, Mark Zuckerberg in Samsung's press conference on February 23, 2016, Fig 1. Someone brings up the internet era will go past, and the era belongs to intelligent robot. As ATM has changed the banking industry, computer has occupied homes and workplaces, intelligent robot and artificial intelligence would be forced many industries to transform in the next 10 years. Intelligent robot will have a profound impact on the economy. By 2025, a driverless car may be by assembled by a machine in the factory without human supervision. People believe intelligent robot can drive well a car than man. Intelligent robot improves not only efficiency and accuracy in health care, but matches gene for each to remedy pertinently. This is a kind of personalized remedy. Hospital will use a robot to give it what it needs in future, whether it is critical for surgery, or care for the elderly and disabled patients ${ }^{[1]}$. Robot maids clean by itself in your House, and your financial adviser which invest automatically for you is a computer.

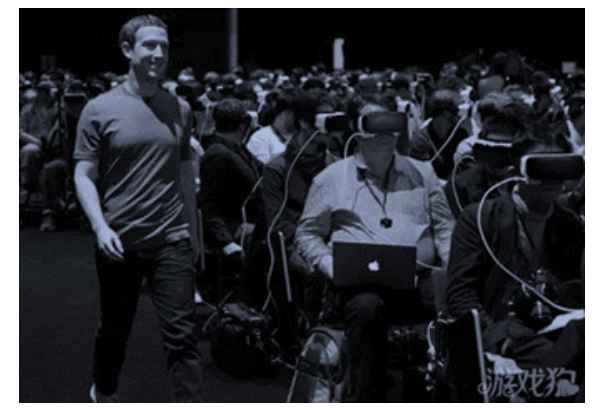

Fig. 1 VR's application in exhibition

AlphaGo comes out a deep shock to humans. It does not replace work on the Assembly line, but any work that can be automated. George Smoot who won the Nobel Prize in physics in 2006 said, $99 \%$ work which has exists at present will be replaced by robots in $20-50$ years ${ }^{[2]}$.

Now, intelligent robot and VR will reform business and services, a profound change in 
technology is bound to change people's way of life. Intelligent robot and AV will change education too. We should think how to use them in education, for it can provide personal and one-to-one's education, which adapt to the requirements of the personality process.

\section{Influence of Intelligent Robots in Education}

In a relatively closed environment, intelligent robot will be more effective than people. Intelligent robot and VR will be an invisible revolution, which communicate seamless in kinds of technology, and it is difficult to be noticed. This will contribute to promoting the interaction between humans and intelligent machines. Further it can also offer multimedia services through actively dialogue--including seeing, hearing, speaking, even much less understand it. Intelligent robot and AR plays a better role in content industry. they have been applied in replying automatically to a message, intellective cha, these applications have entered into the era of the practical application.

Education is a similar environment. People has accessed from the vertical access and applications, analyzed the impact of expert systems, introduced intelligent services to education. Technical monopoly, or example Microsoft, Apple and Google, is studying on intelligent robot to help learning. They hope new technologies to resolve stubborn problems in education, form solutions, and build new productivity tools. they think it is one way to help students perform daily activities. Google has utilized photos identified technologies to help the people finding own lose pet. Uber 's intelligent robot which through the dialogue of people and machines, with Human-centered core requirements, is performed new model by a machine.

Intelligent robot and VR is easy to use in education, for example, some high school students use VR helmet to participate in the opening ceremony in Japan, all of the school's activities are conducted online, fig.2, students come back campuses just a few times in a year.

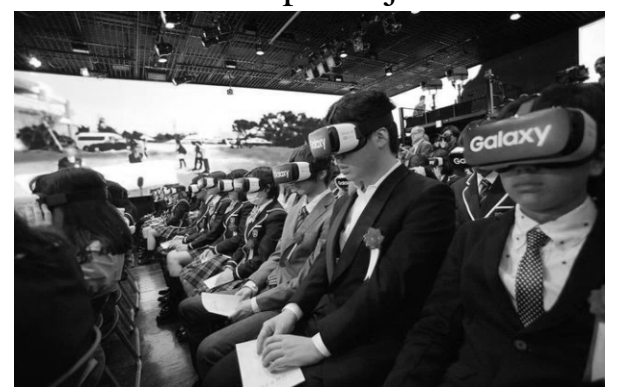

Fig. 2 VR's application in education

Intelligent robot: a crisis, or change? Clearly, Intelligent robot will transform education. It also means that a lot of human crisis. But the human crisis is not necessarily a bad thing. People do not have too many worries, it will bring a better life in future.

First, some history, during the industrial revolution, there have been the process for low wages and middle-class wealth shrink in US. In the short term, intelligent robot and AR may impact on some industries, but they can also bring greater opportunities in other areas in the long run.

Which mechanized equipment is used in agriculture, it saves the human resources and saved the precious time. Intelligent robot improved significantly efficiency in manufacturing industry. In the same way, there are plenty of robot waiter in modern restaurants and hotels. In future, intelligent robot will penetrate into any field of art and the human economy. There is always a different new and often related line of process in different human era, fig 3. 


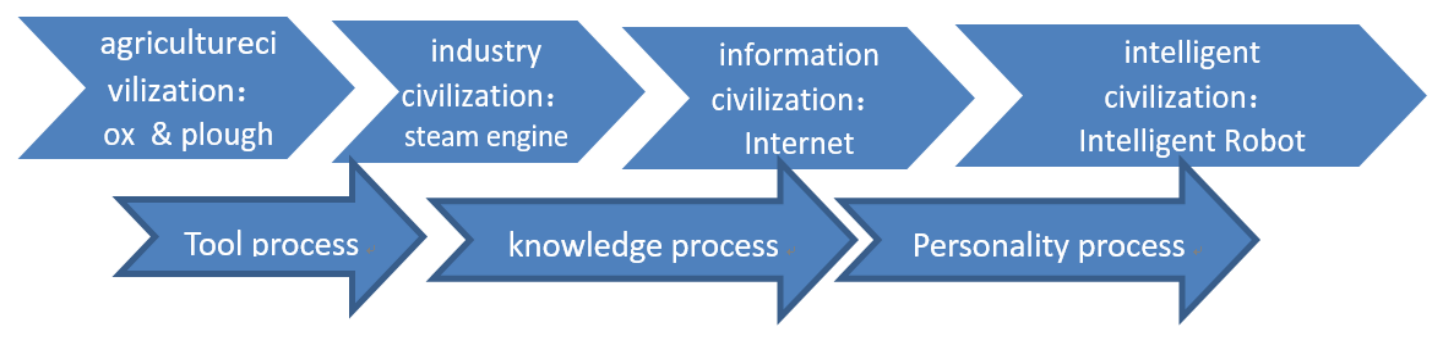

Fig.3 human's process

We are about to enter the era of intelligent civilization; intelligent robot and VR will be a good fit for human. Just like after the industrial revolution, the event which cars replaced carriages, not only improves the efficiency, and brings quite convenient. Therefore, intelligent robot takes over some work, humans are likely to turn to the more creative area, and people's lives better off. On the same, there are also advantages on applying intelligent robot and VR in education:

1.The speed and accuracy and effectiveness of this pattern. AlphaGo has learned about 200,000 high level AndYygo. Its intuition has a solid mathematical Foundation, which can achieve the system's optimization through the mass calculation. This process is scientific and rational judgment, different completely from the human brain, for the human brain is difficult to achieve this level.

2.Big data analytics can be realized. A good trained data can change the industry itself. There has accumulated a large amount of data in education, with the help of data analysis, human can obtain quickly educational experience, and soar to any corner of the world by network.

3.Services more widely. A good intelligent robot can serve more people. In the movie "she", OS1 communicate with 8,316 peoples in the same time, and the hero Theodore is just one of them. This illustrates a robot can serve plenty of students well.

4.Personalized service. As in the movie "she", the heroine Samantha is considerate and humorous, and her rate of study and evolution was amazed the hero Theodore, they both quickly become no don't talk of friend. Theodore found that a few classmates congenial and desires, human fall in love with machine without worldly understanding.

\section{Applying Patterns of Intelligent Robots in Education}

Which intelligent robot combines VR, it can be integration some sensory experiences, improves student's experience and understand in new ways.

1. A virtual learning platform. Intelligent robot can provide a digital environment which can connect to reality. In this digital environment, it delivers different kinds of learning content to any student according to each student's demand. But education is the model of industrial age in today, a group of children were herded into "educational factory", without regard as their individuality, and sharp them according to the same content. A digital environment which can connect to reality which integrate of virtual material. It will make traditional education services become a perfect gathering integration, including of mobile Internet, $\mathrm{AR}$, wearable device and so on. It will achieve each individual's talents. A good significance teaching is superior to lists of data at all times, for it is easier to accept.

2. A virtual research platform. On special situation, for example, volcano outbreak, cell split and ancient biological and so on, there are some dangerous or not easy reach, even simply did not exist. Intelligent robot can construct a "real" of learning stories; multimedia display can help knowledge of assimilation. Compared to text form of rendering of information, visual rendering realization of dynamic simulation and stereoscopic visual simulation can usually more effect.

3. A cooperative work platform. Intelligent robot is an excellent scientific research platform. 
Student's learning is not just the process of acquiring new skills, also a social behavior which people share a common habit. Cooperative learning can obtain better reach in groups. If knowledge is embedded in the social interaction team, it will become community of practice.

4. A Test, Results Discussion and Prediction platform. Intelligent robot has great appeal to teenagers. Most children like to play with robot. As we all know, interest is the best teacher, as long as the child is interested in it, which would have a strong motivation and change from passive to active. More generally, intelligent robot has the ability to perceive information in itself and the environment, self-acting achieves a pre-determined set of objectives and tasks, dynamically trace a real-time monitoring process, and analyze student's behavior of learning.

\section{Existing problems and Solutions in Intelligent Education}

But there are some problems in education yet now.

1. Fully functional robots is difficult to achieve. We should reconstruct its function. AS OSI (a kind of networking protocol) is divided into 7 layers, which is not only easy to achieve, and easy to upgrade and standardize. Using this pattern, Intelligent robot will greatly improve its application and popularization in education.

2. It is difficult to distinct digitization and intelligence. AlphaGo prevails over Lee Se-dol, does not mean that intelligent robot really smart as human beings. The game of Go is easy to digitization, but it is not intelligence.

3. It do not replace the value of a human. People should deal properly with relation of digital \& real, natural thinking \& machine intelligence. A robot, which can think as human do, is hard to come into being in a short time. Just like Ian Pearson said:" you can't really say, you can find any information what you want by Google, you are no longer need teachers". Robots are unable to get along with real people, for human emotions cannot be processed.

4. Financial problem. About 4 billion people cannot access to Internet in the world. There is more serious in rural areas of Asia and Africa. How to benefit rural people by technology? We should develop infrastructure which access easy to the Internet, it will bring a great deal of help on poverty reduction.

\section{Conclusion}

We can draw some conclusions.

1. A widespread use of intelligent robot in education. The application of intelligent robot is almost certain to beneficial to students' learning. With continuous improvement of intelligent robot, it can bring up more powerful information, user's experience will also get better and better. There is no doubt, intelligent robot has the power to change the way we work. When student no work pressure, they can enjoy a more interesting and visionary lives.

2. The rapid development and great changes in education. Technical invention is the catalyst for social development. A breakthrough invention can lead to a series of new inventions, more profound change. The wheel of history cannot be prevented, the key is how to adapt. In modern education, even if intelligent robot cannot change the essence of educational process, it will change the organization sequence of educational process, influence ideas how to solve education problems, make the mode leap and raise the effect of teaching. 


\section{Reference:}

[1]. www.robot-china.com. Nobel Prize winners on Shen Yang: robots will substitute for 99\% work [OL]. http://www.robot-china.com/news/201509/01/24676.html. 2015. 09 .01(In Chinese).

[2]. OF week Robots. Robots era: 27 future prophecies on 8 major industries [OL].

http://robot.ofweek.com/2016-03/ART-8321202-8500-29072185.html. 2016. 03 .04(In Chinese). 